\title{
A REVIEW ON MICROSPHERES AS A NOVEL CONTROLLED DRUG DELIVERY SYSTEM
}

\author{
MOUSAMI S SAMANTA*, DEEPAK GAUTAM, MUHAMMED WASIM CHANDEL, GAURANG SAWANT, KIRTI SHARMA
}

Department of Pharmaceutics, H.K. College of Pharmacy, Oshiwara, Mumbai, Maharashtra, India. Email: mausam39229@gmail.com

Received: 26 December 2020, Revised and Accepted: 13 February 2021

\begin{abstract}
Over the past three decades, controlled drug delivery systems have become more developed and play a key role in pharmaceuticals formulations. There are many shortcomings in Traditional or Conventional drug delivery systems like for maintaining desired therapeutic drug plasma concentration there is a need for frequent dosing for particular drugs having shorter half-lives. Furthermore, because of frequent dosing requirement, there is poor patient compliance which causes fluctuation in plasma concentration of the drug. The limitations of conventional drug delivery can be overcome by the development of novel drug delivery systems, of which the controlled drug delivery can maintain constant drug plasma concentration by slowly releasing the drug over an extended period. Developing controlled drug delivery systems can also improve the systemic bioavailability of the drug, thus enhancing the therapeutic efficacy of the drug and better patient compliance. There are many different approaches for such controlled delivery systems such as liposomes, niosomes, ethosomes, phytosomes, microemulsion, and microspheres. Among all the approaches microspheres are more convenient as the drug is slowly released from the polymeric matrix and the polymers used are mostly biodegradable and possess no side effects. Therefore, microspheres can be used in various medicinal departments such as oncology, gynecology, radiology, pulmonary, cardiology, diabetes, and vaccine therapy. This review article focuses on recent different types of microspheres along with their methods of preparation. The microspheres formulated can be later evaluated and characterized by different procedures.
\end{abstract}

Keywords: Controlled drug delivery system, Improved systemic bioavailability, The constant drug plasma level.

(C) 2021 The Authors. Published by Innovare Academic Sciences Pvt Ltd. This is an open access article under the CC BY license (http://creativecommons.org/ licenses/by/4.0/) DOI: http://dx.doi.org/10.22159/ajpcr.2021v14i4.40634. Journal homepage: https://innovareacademics.in/journals/index.php/ajpcr

\section{INTRODUCTION}

Over $90 \%$ of the existing therapeutics is administered through the most preferable and convenient route, that is, the oral route of drug administration. Whenever a New Chemical Entity is discovered the first challenge that stands to the pharmaceutical companies is how to formulate the dosage form so that it can be effectively administered by oral route in the first place. However, the conventional oral drug delivery systems have a limitation of poor therapeutic efficacy due to frequent dosing of drug to achieve constant plasma concentration and poor patient compliance. Such pharmacokinetic limitations can be overcome by improving the dosage form to release the drug slowly in a controlled manner over an extended period of time. One such approach is microspheres also known as microparticles [1]. Microspheres are characterized as spherical microparticulate and free-flowing powders consisting of biodegradable polymers mostly. They ideally have a particle size ranging from $1 \mu \mathrm{m}$ to $1000 \mu \mathrm{m}$. Microspheres can be loaded with drug and use for targeted drug delivering. As the drug is loaded in polymeric microspheres, it shows therapeutic action on targeted tissue only. Microspheres are designed to enhance the therapeutic effectiveness of the drug and achieve better bioavailability thereby minimizing the toxicity and minimal side effects [2].

There are two types of microspheres, reservoir type, and matrix type.

\section{Reservoir types}

In this system, the drug is entrapped as a core inside a water-insoluble polymer, which controls the rate of drug release. The commonly used polymers in such devices are ethylcellulose or polyvinyl acetate. This type is also known as microcapsules.

\section{Matrix types}

In this type, the drug is homogeneously distributed in a polymeric matrix, which controls the drug release rate. The commonly used polymers for matrix types are sodium alginate or hydroxypropyl methylcellulose (HPMC). This type is also known as micromatrices [3].
There are two mechanisms of drug release from the microspheres

1. Dissolution: Here, the drug dissolution rate is controlled by the polymer decreasing its wettability or by itself getting dissolve in GI fluid at a slower rate.

2. Diffusion: Here, the drug diffuses from a region of higher concentration to the lower concentration of drug [6].

\section{ADVANTAGES}

\section{Better patience compliance}

As microspheres provide a slow release of drug for an extended period of time, there is a reduction in dosing frequency due to which it is better for patient compliance mainly pediatrics, geriatrics, psychology patients, etc.

\section{Enhance bioavailability}

Microspheres are micron in size, that is, less size give more surface area to increase the solubility of poorly soluble drugs and hence increasing the systemic bioavailability of the drugs.

\section{Constant drug plasma concentration}

Microspheres show a controlled release of drug for a prolonged time; as a result, there is no the fluctuation of drug concentration in systemic circulation and a constant $\mathrm{C}$ max is achieved.

\section{Reduction in adverse effects}

Biodegradable polymeric microspheres are biocompatible with body environment; they do not require to be removed surgically. As the drug is controlled released, the systemic toxicity is also reduced.

\section{Enhance stability}

Liquid drugs can be converted into solid microspheres to increase the stability of the drug and also for maintaining its clinical shelf life.

\section{Parenteral formulation}

Microspheres are spherical in shape a high dose of the drug can be given as microsphere parental depot. 
Targeted drug delivery

Microspheres are used to target disease sites specifically tumor tissues, while the concentration remains low at remaining normal tissues $[4,5]$.

\section{DISADVANTAGES}

\section{Production cost}

The cost of manufacturing of controlled drug dosage form is higher than the cost of manufacturing of conventional dosage forms.

\section{Reproducibility}

Microspheres are hard to reproduce as specialization and technologies are required for manufacturing microspheres.

\section{Potential toxicity}

As microspheres are loaded with a high concentration of drugs, there are chances of dose dumping leading to potential toxicity.

\section{Polymeric toxicity}

Polymeric additives such as plasticizers, stabilizers, and antioxidants are also used depending on the formulation design these polymers can undergo hydrolysis, oxidation, or react to biological agents causing toxicity.

\section{Swallowing}

The microsphere intended for oral administration should be swallowed and not chewed or crushed because they are designed for prolonging the release of drugs.

\section{Maintaining conditions}

The processing conditions of microspheres such as $\mathrm{pH}$, temperature, agitation, solvent evaporation, and heating can influence the stability of the drug to be encapsulated $[4,5]$

\section{TYPES OF MICROSPHERES}

\section{Bioadhesive microspheres}

Microspheres loaded with drug adhering to the mucosal layer present in buccal, ocular, rectal, nasal, etc., using the adhering property of the water-soluble polymers are known as bioadhesion. These kinds of microspheres show properties such as close exposure to the absorption area. It also shows extended residence time at the targeted area and hence, produces a better therapeutic efficacy, for example, ophthalmic administration of Acyclovir, nasal administration of insulin, and buccal administration of nifedipine through bioadhesive microspheres $[7,8]$.

\section{Magnetic microspheres}

This type of microsphere has an important property to use as a conveyance system, that is, it restricts the drug to the disease site. The main focus of these kinds of microspheres is on replacement of high quantity of freely disseminating drug with a less quantity of the magnetically targeted drug. Magnetic microspheres are of size $<4 \mu \mathrm{m}$, due to which it adequately circulates the blood capillaries without any vascular occlusion and are targeted to disease site through an external magnetic field of 0.5-0.8 tesla, for example, Removal of neuroblastoma cells from bone marrow with monoclonal antibodies conjugated to magnetic microspheres $[9,10]$.

Magnetic microspheres are of two types:

\section{Therapeutic magnetic microsphere}

The main focus of this kind of microsphere is targeting liver tumors by transporting a chemotherapeutic agent to the disease site. These types of microspheres are mainly loaded with proteins or peptide drugs for targeting.

\section{Diagnostic magnetic microsphere}

These types of microspheres mainly focused on liver metastases imaging. It can also be used for composing nano-sized particles like supramagnetic iron oxides which are used to differentiate bowel loops from other abdominal structures.

\section{Floating microspheres}

The floating microspheres have a bulk density which is less when compared to gastric fluid because of which, without influencing gastric emptying rate it remains floating in the stomach. In this release of the drug takes place gradually at the desired rate, this type of microspheres found to be buoyant on gastric content and increases gastric residence time leading to a constant level of drug plasma concentration. Because of this kind of microspheres rate of dosing frequency also reduces and it also produces an extended therapeutic effect, for example, Ketoprofen floating microspheres and Felodipine floating microspheres [11,12].

There are two types of floating microspheres:

1. Effervescent microspheres

2. Non-effervescent microspheres.

\section{Radioactive microspheres}

The size range of radioembolization therapy microspheres is $10-30 \mathrm{~nm}$ which is greater than the diameter of the capillaries and is tapped in the first capillary bed. These microspheres are injected in the arteries leading to the targeted tumor tissues.

So in all these situations radioactive microspheres without harming remaining tissues focus on a particular site and transport high radiation dose. The various types of radioactive microspheres are $\alpha$ emitters, $\beta$ emitters, and $\gamma$ emitters [13].

\section{Polymeric microspheres}

The various types of polymeric microspheres can be classified as follows.

\section{Biodegradable polymeric microspheres}

Starch which is a natural polymer is used in microsphere formulation; it is biodegradable, biocompatible, and bioadhesive in nature. These kinds of biodegradable polymers show extended residence time when coming in exposure with mucous membrane because of its potency of swelling property due to contact with an aqueous medium, finally resulting in gel formation. The rate and amount of drug which is released from the microsphere totally depend on and is controlled by the concentration of polymer and the release pattern. This release pattern takes place in a sustained manner. The main disadvantage of biodegradable microspheres is that its drug loading efficiency and drug release. However, they give a broad range of application in microspherebased treatment, for example, Polylactic acid microspheres loaded with 5 -fluorouracil.

\section{Synthetic polymeric microsphere}

These types of microspheres are widely employed in clinical utilization, additionally also are useful as a bulking agent, fillers, embolic particles, drug delivery medium, etc., and are demonstrated to be safe, biocompatible but the main drawback of these type of microspheres, are they shift away from the injection site and causes possible risk, embolism, and further organ damage, for example, Phenobarbitone microspheres using polymer Eudragit RL [14].

\section{Porous microspheres}

Porous microspheres either have external surface pores or internal pores in the core where the active pharmaceutical ingredient can be dispersed or dissolved as shown in Fig. 1. The pores are formed by porogens which leach out completely later in process, for example, of porogen used is effervescent salts such as ammonium bicarbonate, hydrocarbon waxes, inorganic salts such as sodium chloride, carbohydrates, ice, linear polymers, gelatin, and sugar. The outer framework is fabricated with materials such as calcium carbonate $\left(\mathrm{CaCO}_{3}\right)$, mesoporous silica, hydroxyapatite, and biodegradable porous starch foam, for example, delivery of proteins and peptides $[15,16]$.

\section{Glass microspheres}

Hollow glass microspheres are finely dispersed free-flowing novel form of glass material consisting of a $10 \mu \mathrm{m}$ to $100 \mu \mathrm{m}$ diameter with a hollow 


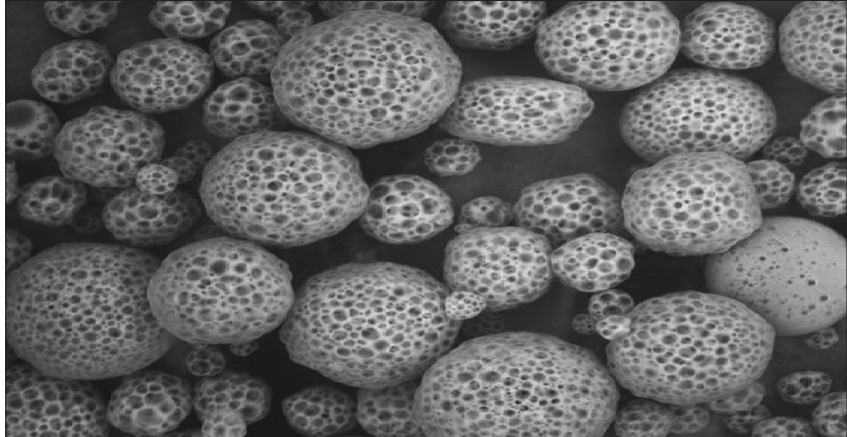

Fig. 1: Porous microspheres as observed under the scanning electron microscope. Adapted from the Koch Institute Public Galleries (https://ki-galleries.mit.edu/2015/tzeng). Adapted by permission from The Koch Institute of Integrative Cancer Research at Massachusetts Institute of Technology (Research Institute at Cambridge, United States of America)

central cavity surrounded by a $1 \mu \mathrm{m}$ thick silica shell, for example, hollow glass microspheres are potential nanocarriers for oligonucleotides and proteins in tissue engineering. However, drug delivery applications require either an organic copolymer or incorporation of materials (such as metal ions) within the glass matrix $[17,18]$.

\section{INGREDIENTS OF MICROSPHERES}

\section{Polymers}

In microsphere formulation, most commonly biodegradable and non-biodegradable variety of polymers are used by researchers. The polymers which are used in microspheres preparation are classifies into different types as Natural and Synthetic. Before choosing the polymer for the microsphere formulation, we need to consider a few parameters such as nontoxicity, biocompatibility, biodegradability, and easy availability of polymers. It should be biocompatible, biodegradable, non-toxic, and easily available. These polymers which pass all parameters for their selection have many advantages like they increase the residence time of the drug in the body because of which we get better bioavailability of drug compared to conventional drug delivery system. Examples of natural polymers include Albumin, Collagen, and Gelatin which are proteins while Agarose, Carrageenan, Chitosan, and Starch are carbohydrates whereas Poly (acryl) Dextran, Poly Starch, and DEAE Cellulose are chemically modified carbohydrates and Sodium Alginate, cellulose ether, xanthan gum, Scheroglucan, Gum Arabica, Tamarind seed polysaccharide, Beeswax, carnauba wax, Chitin, and Corn protein (Zien) form the other class. Examples of synthetic polymers fall under three category where Lactides, Glycolides and their copolymers, polyanhydrides, poly alkyl cyanoacrylates are biodegradable in nature while Glycidyl methacrylate, Acrolein, Epoxy polymers, and polymethyl methacrylate are non-biodegradable and finally, polysebacic anhydrides, Poly Esters/Poly Lactides, poly orthoesters, polycarbonates, polylactic glycolic acid (PLGA), polycaprolactones, polyphosphazenes, ethylcellulose, Eudragit L100, Eudragit S100, HPMC, Eudragit RS100, and Eudragit RL100 form the other class [19-24].

\section{Surfactant}

In microsphere formation surfactant play an important role during emulsification and extrusion process. Surfactants play an important role by lowering the interfacial tension between hydrophilic and hydrophobic molecules, because of which stable emulsion is formed. Use of surfactant leads to the formation of discrete microspheres by preventing the emulsion droplets from coalescing. Hydrophiliclipophilic balance (HLB) indicator is used for selection of proper emulsifier. Hydrophilic surfactants have HLB value in the range of 8-18 and are used for oil in water emulsion while emulsifiers with HLB value in the range of 3.5-6 are known as lipophilic surfactants. The particle size of microspheres is decreased by increasing the concentration of surfactant because of which smaller size and size distribution of microspheres were formed. Examples are Sodium Laureth Sulfate, Sodium dodecyl sulfate as anionic surfactants and Polysorbate 80, Tween 40, Tween 20, Span 85, Span 80, Span 20, Poloxamer188, Brij58, Poly Glycerol Polyricinoleate, and Sorbitan as non-ionic surfactants [25-31].

\section{Oil}

Particle size, size distribution, and uniformity of microspheres are affected by the ratio of viscosity of the oil phase to the viscosity of the water phase, for example, It is reported that the particle size of microspheres are more which were prepared using olive oil compared to microspheres which were prepared using liquid paraffin as the viscosity of olive oil is higher compared to liquid paraffin oil. There are various types of oils which are used in the fabrication of microspheres during emulsification/gelation method. Examples are liquid paraffin, soya bean oil, olive oil, sunflower oil, castor oil, groundnut oil, rapeseed oil, and rapeseed methyl esters [32].

\section{Crosslinkers}

Most commonly used crosslinkers for microspheres preparation are $\mathrm{Ca} 2+, \mathrm{Sr} 2+$, and $\mathrm{Ba} 2+$ ions. However, $\mathrm{Sr} 2+$ and $\mathrm{Ba} 2+$ ions are mildly toxic and $\mathrm{Ca} 2+$ ions are non-toxic because of which $\mathrm{Ca} 2+$ ions are widely used crosslinkers for preparation of microspheres. At low concentration of $\mathrm{Ca} 2+$ ions agglomeration of microspheres takes place. By increasing $\mathrm{Ca} 2+$ ions concentration entrapment efficiency of microsphere slightly increases. However, after optimum concentration of cross-linker if more crosslinker is added the entrapment efficiency decreases due to overloading of crosslinker. Examples are glutaraldehyde, sulfuric acid, and calcium carbonate [33-36].

\section{Solvent}

Solvents mostly used when microspheres are prepared using a solvent evaporation method. Examples are Chloroform, Dichloromethane (DCM), Ethanol, Acetonitrile, Polyvinyl alcohol (PVA), Methylene chloride, and Methanol [37-39].

\section{METHODS OF PREPARATION}

\section{Spray drying}

In this technique coating polymer is first to dissolve/dispersed in an organic solvent such as acetone and DCM the drug then incorporated into polymeric solution along with high-speed homogenization [40]. The resultant mixture then atomized in a stream of hot air. Atomization leads to the formation of fine mist or droplets from which organic solvent evaporates immediately which leads to the formation of microspheres in a size range of $10 \mathrm{um}-100 \mathrm{um}$ [41].

\section{Solvent evaporation}

This method involves the use of organic phase as manufacturing vehicle; this process consists of two phases. First is the aqueous phase in which the drug is incorporated along with the stabilizing agent or without stabilizing agent. Moreover, the other phase is organic phase consists of polymer solution in an organic volatile solvent such as acetone and DCM then aqueous and the organic phase should be mixed with high-speed homogenization which leads to the formation of $\mathrm{w} / \mathrm{o}$ emulsion then this emulsion is added in the large aqueous phase to form w/o/w emulsion if necessary. The resultant mixture heated along with continuous stirring which leads to evaporation of the organic phase which leads to shrinking the coating polymer across the core material and leads to the formation of microspheres [40].

\section{Single emulsion technique}

In this method, microsphere is prepared by emulsification technique; coating polymer is dissolved in an organic volatile solvent which leads to the formation of a polymeric solution. The resultant polymeric solution added into an aqueous phase containing emulsifying agent leads to the formation of o/w emulsion. This emulsion is then stirred for a few hours under constant environmental condition, then filtered, and dried into desiccator [42]. 
Double emulsion technique

This technique involves the preparation of double emulsion either $\mathrm{w} / \mathrm{o} / \mathrm{w}$ or o/w/o type. The Aqueous solution contains the drug which is dispersed in the organic phase. The organic phase containing coating polymer encapsulates the drug present in the dispersed aqueous phase and leads to the formation of primary emulsion. Then, this primary emulsion undergoes homogenizing or sonicating before adding into an aqueous solution of PVA to form a secondary emulsion, and then prepared microspheres filtered and dried in desiccator [43].

\section{Phase separation coacervation technique}

This method is generally used for fabrication of reservoir type of microspheres. Mostly this method used to encapsulate the hydrophilic drugs; in this method, coating polymer is dissolved in an organic volatile solvent and then an aqueous solution of the drug is added to allow the polymer to coat drug, then phase separation will be initiated by changing the ambient conditions such as changing temperature, changing $\mathrm{pH}$, and the addition of salt [44].

\section{Spray congealing}

In this technique, the drug is dissolved/dispersed in polymeric solution, that is, lipophilic polymer like wax. The hot molten solution then sprayed to form fine droplets into a vessel that already kept in carbon dioxide ice bath [45].

\section{Solvent extraction}

This method involves the removal of the organic phase by extraction of the organic solvent through using hydrophilic organic solvents like isopropyl-alcohol. Organic phase then extracted using water, this process leads to a decrease in the hardening time of microspheres [46].

\section{Quassi emulsion solvent diffusion}

Using this technique microsponge could be prepared. It involves two phases one is internal and the other is external. The external phase consists of PVA and distilled water and internal phase consist of polymer, drug, and ethanol. Internal phase is heated up to $60^{\circ} \mathrm{C}$ and then added to the external phase main. It is then maintained at room temperature. Resultant emulsion is then homogenized up to $2 \mathrm{~h}$ and fabricated into microsponges then filtered, washed, and dried in a vacuum oven for $24 \mathrm{~h}$ [47].

\section{Cross-linking agent method}

In this method, cross-linking agent is used for fabrication of the microspheres. The first specific concentrated polymeric solution has been made in an aqueous medium then added in continuous phase containing oil and specific concentration of surfactant to form w/o emulsion, followed by drop by drop incorporation of an aqueous solution of cross-linker coupled with continuous agitation and then permitting for the stiffening of the surface of microspheres. Resultant microspheres then washed and dried [48].

\section{Hot melt microencapsulation}

In this technique, coating polymer is melted and then homogenized with the drug, the resultant mixture is then suspended in a lipophilic solvent such as silicon oil along with continuous agitation/stirring and heating the solution at $5^{\circ} \mathrm{C}$ up to the melting point of the polymer after the emulsion get stabilized, it is cooled to solidify the polymeric microspheres [49].

\section{Ionic gelation method}

In this method, suspension of hydrophilic polymer along with drug is complexed with multivalent cation, that is, calcium chloride resulting in the formation of highly viscous gel spheres as shown in Fig. 2. An iridescent suspension is obtained. This suspension is centrifuged to get the uniform size of microspheres. Microspheres are then washed and dried at room temperature for $24 \mathrm{~h}[50,51]$.

Hydroxyl appetite (HAP) microspheres in sphere morphology Microspheres were fabricated by o/w emulsion followed by evaporation of the organic solvent. The first organic phase (drug-containing 5\% w/w

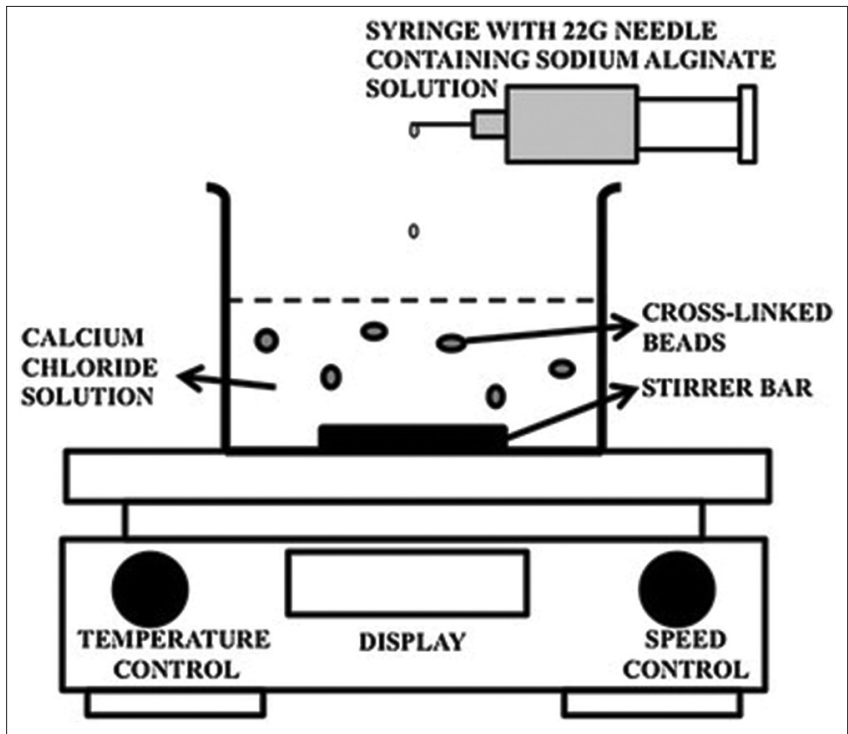

Fig. 2: Schematic illustration of the ionic gelation method. Adapted from reference [30]. Copyright permission obtained under creative commons (CC BY) license. (http:// creativecommons.org/licenses/by/4.0/)

of EVA and a suitable amount of HAP) is dispersed in the aqueous phase of surfactant to form o/w type emulsion. The organic phase is dispersed in tiny droplets surrounded by surfactant moieties which prevent the droplets to recombine and helping them to stay discrete droplet, while thrilling the DCM started evaporating slowly leave behind the droplets of microspheres [52-54].

\section{EVALUATION TECHNIQUES}

\section{Characteristics}

It is an important phenomenon to characterize these microparticulate carriers, which helps design a sustainable and suitable carrier for proteins, drug, or antigen delivery. Every microsphere has different microstructures. These microstructures are determining the release and stability of the carrier.

\section{Particle size and shape}

The most common ways to picture microspheres are conventional light microscopy (LM) Confocal fluorescence microscopy and scanning electron microscopy (SEM). These techniques can be used to determine the shape and outer structure of microspheres. Conventional LM: Conventional LM provides control over coating parameters in the case of double-walled microspheres. The microsphere's structures can be visualized before and after coating and the change can be measured microscopically. SEM provides higher resolution in contrast to LM. SEM: SEM allows investigations of the surfaces of the microspheres and after particles are cross-sectioned, it can also be used for the investigation of double-walled systems [59]. Confocal fluorescence microscopy: Confocal fluorescence microscopy is used to characterize the structure of multiple walled microspheres. Laser light scattering and multi-size coulter counter other than instrumental methods, which can be used for the characterization of size, shape, and morphology of the microspheres [55].

\section{Angle of contact}

The angle of contact determines the wetting property of microspheres in terms of hydrophobicity and hydrophilicity. This thermodynamic property is specified to a solid substance and affected by the presence of an absorbed component. The angle of contact is measured at solid/ air/water interface. The increasing and decreasing angle of contact are measured by placing a droplet in a circular cell mounted above the objective of an inverted microscope. The contact angle is measured at $200^{\circ} \mathrm{C}$ within a minute of deposition of microspheres [57]. 
Attenuated total reflectance Fourier transform infrared (FT-IR) spectroscopy

FT-IR determines the degradation of the polymeric matrix of the carrier system and the surface of the microspheres is determined by calculating the alternated total reflectance (ATR). A beam of infrared light is passed through the ATR crystal in such a way that it reflects many times through the sample to provide IR spectra mainly of surface material. Together ATR-FTIR provides information about the surface composition of the sample microspheres [57].

\section{Density determination}

The multi-volume pycnometer is used to determine the density of microspheres. In a cup accurately weighed sample of microspheres is placed and then the cup is placed in the multi-volume pycnometer in a cup is placed into the multi-volume pycnometer. At constant pressure, helium gas is introduced in the chamber and allowed to expand. Expansion of the helium gas decreases the pressure within the chamber and two consecutive readings of reduction in pressure at different initial pressure are noted. From these two pressure readings, the volume and the density of the sample microsphere are determined [57].

\section{Electron spectroscopy for chemical analysis (ESCA)}

ESCA determines the surface chemistry of the microspheres. ESCA also determines the atomic composition of the surface of microsphere and surficial degradation of the biodegradable microspheres by creating spectra by the help of electron spectroscopy. It is also called also called X-ray photoelectron spectroscopy [57].

\section{Surface carboxylic acid residue}

Radioactive glycine is used to measure surface carboxylic acid residue. Radioactive glycine conjugates are synthesized by the chemical reaction between c14 - glycine ethyl ester hydrochloride and the sample microspheres. The glycine residue formed is linked using the water-soluble condensing 1- ethyl-3 (3-dimethyl aminopropyl) carbodiimide (EDAC). Radioactivity of the conjugate is then measured using a liquid scintillation counter technique. Thus, the carboxylic acid residue can be compared against the standard and inference drawn accordingly. Free carboxylic acid residue can be used to measure hydrophobic or hydrophilic or any other derivatized type of the microspheres [57].

\section{Isoelectric point}

To determine isoelectric point an apparatus known as micro electrophoresis is used that measures the electrophoretic mobility of microspheres. Mean velocity at each $\mathrm{pH}$ values ranging from 3 to 10 is calculated by measuring the time of particle movement over a distance of $1 \mathrm{~mm}$. Electrical mobility of the particle can be determined using this data. The electrophoretic mobility can be related to these three parameters such as surface contained charge, ionizable behavior or ion absorption nature of the microspheres [57].

\section{Surface amino acid residue}

The radioactive c14-acetic acid conjugate is used in the determination of surface associated amino acid residue. The amino acid residue is determined indirectly by first determining carboxylic acid residue through liquid scintillation counter. EDAC is used to condense the amino group and the c14 - acetic acid carboxylic acid residue. The indirect estimation method is done to determine the free amino or the free carboxylic acid residues, by measuring the radioactivity of the c14 glycine ethyl ester hydrochloride having acetic acid or the glycine conjugates [57].

\section{Capture efficiency}

Capture efficiency of the microspheres is determined by allowing washed microspheres to the lysate. The lysate is then tested as specified in the particular monograph for the calculation of the active constituents present in the formulation. The percent encapsulation efficiency is calculated using the following equation: \% Entrapment=Actual content/Theoretical content $\times 100$ [57].

\section{In vivo methods}

In vivo methods are methods for studying the permeability on intact mucosa. These techniques exploit the biological response of the organism locally or systemically. Some of the earliest and simplest studies of mucosal layer permeability were determined by the systemic pharmacological effects produced by drugs after ingestion or absorption into the oral mucosa. However, now the most widely used methods are using animal models, buccal absorption tests, and corneal perfusion chambers for studying drug permeability [4].

\section{Animal models}

A series of compounds are screened using animal models to investigate the mechanisms and usefulness of permeation enhancers or evaluating a set of formulations. Several animal models such as the dog, rats, rabbits, cat, hamster, pigs, and sheep have been known. The procedure involves anaesthetizing the animal followed by the administration of dosage form for which the study has to be done. The esophagus of rats is ligated to prevent absorption pathways other than an oral mucosal layer. The absorption rate is determined by withdrawing blood at different time intervals and analyzing [4].

\section{Buccal absorption test}

It is known for its simplicity and reliability for measuring the extent of drug loss in the oral cavity through single and multi-component mixtures of drugs. Through this test method, the structure, contact time, $\mathrm{PH}$, and initial drug concentration of the solution is determined when the drug is held in the oral cavity [61].

\section{Corneal perfusion chambers}

In the development and assessment of ophthalmic drugs, corneal perfusion chamber method is termed very useful. This study aims to design and test a modified perfusion chamber which is suitable for topical application of drugs that are isolated to corneoscleral preparations, and which allows continuous monitoring of endothelial cell function. In this method, a perfusion chamber is designed out of polycarbonate and stainless-steel to clamp corneas in a horizontal plane which makes it suitable for topical drug delivery. The endothelial cell function was assessed by ultrasonic pachymetry and specular microscopy during this perfusion. Epithelial barrier function was assessed by the amount of penetration of fluorescein. Leakage was examined by measuring the penetration of a large protein. By conventional histology tissue architecture after perfusion was examined [56].

\section{In vitro methods}

\section{Beaker method}

In this method, the dosage form is made to adhere at the bottom of the beaker containing the medium and stirred uniformly using an overhead stirrer. The quantity of the media employed ranges from 50 to 500 $\mathrm{ml}$ and the stirrer speed form 60 to $300 \mathrm{rpm}$. A sample is withdrawn in time intervals and the amount of drug dissolved in the medium is determined [60].

\section{Interface diffusion system}

Interface diffusion system method was developed by Dearden \& Tomlinson. It comprises four compartments. Compartment A represents the oral cavity which contains an appropriate concentration of drug in a buffer. Compartment B representing the buccal membrane, containing 1-octanol, and compartment $\mathrm{C}$ is representing body fluids, which contains $0.2 \mathrm{M} \mathrm{HCl}$. The compartment D representing protein binding which also contains 1-octanol. Before use, the aqueous phase and 1-octanol were saturated with each other. Samples were withdrawn and returned to compartment A with a syringe. Hence, the drug dissolved in different cavities of the human body is determined by analyzing samples from all four compartments [4].

\section{Modified keshary chien cell}

A specialized apparatus was designed in the laboratory. It consisted of a Keshary Chien cell which contains distilled water $(50 \mathrm{ml})$ at $370^{\circ} \mathrm{C}$ as the 
dissolution medium. Mostly all Trans Membrane Drug Delivery Systems are kept in a glass tube attached with a 10\# sieve placed at the bottom which is then reciprocated in the dissolution fluid at a rate of 30 strokes/min thus, determining the rate of dissolution of the drug delivery system [60].

\section{Dissolution apparatus}

Standard USP or BP dissolution apparatus is used to study in vitro drug release profiles using both the rotating elements which are paddle 41, 42,43 and basket 44,45 . Dissolution medium which is used for the study varies from 100 to $500 \mathrm{ml}$ and the speed of rotation varies from 50 to $100 \mathrm{rpm}[60]$.

\section{Other methods}

Few other methods involve plexiglass sample blocks placed in flasks 46, agar gel method 47, etc., have also been reported. Although several methods have been reported, the ideal method would be one where sink condition is maintained and dissolution time in vitro simulates dissolution time in vivo [4].

\section{APPLICATIONS OF MICROSPHERES IN PHARMACEUTICAL INDUSTRY}

\section{Oral drug delivery}

The oral route is an easy and convenient route for administration of the drug with higher patient compliance. There are large numbers of pharmaceutical products administered through the oral route. The principal behind oral absorption totally depends on the solubility and permeability of the drug. Microsphere drug delivery offers a sustained and controlled manner drug release for a longer period of time leads to reduce dosing frequency and improve patient compliance [62].

\section{Ocular drug delivery}

Microspheres are a good carrier for the ocular drug delivery. Using microspheres drug delivery bioavailability of the drug has been improved as compared to the aqueous ocular preparations. Due to their sustained or controlled release mechanism microspheres are used for the longlasting release of drug which leads to reduce the dosing frequency [63].

\section{Intranasal drug delivery}

This route is mainly preferred for the delivery of proteins and the peptides. Conventional formulations are easily get drained off from nasal mucosa. Bioadhesive microspheres provide better bioavailability by exerting its sustained/controlled mechanism [64].

\section{Gene therapy}

In this technique, microspheres are fabricated with viral vectors in genedrug delivery. This technique offers ease of preparation, site targeting, and large scale production and shows low immunogenic response as compared to the direct viral vector drug delivery [65].

\section{Buccal drug delivery}

Mucoadhesive microspheres serve as a reservoir for the drug; it releases the drug from the applied site for a longer period of time. Mucoadhesive polymers reside on the mucosa of the buccal cavity and act as a reservoir; it also improves the bioavailability of the drug by avoiding first-pass metabolism in the body [62].

\section{Transdermal and topical drug delivery}

Polymers having good film-forming ability used for delivery of drug through the skin, for example, Chitosan, Alginate, and PLGA loaded microspheres are used as Transdermal Drug Delivery. It also used for delivering the drug for topical application, for example, Asiaticoside loaded microspheres for wound healing showing an acceleration in reepithelization as well as promoting the angiogenesis [66].

\section{Gastrointestinal drug delivery}

Microspheres are used for delivery of the potent drug to the specific site (Gastrointestinal tract [GIT]).

Eudragit, ethylcellulose, carbopol, and alginate microspheres are used for delivery of the drug at a specific site in the GIT. It prevents the first pass hepatic metabolism of the drug and increases the bioavailability of the drug [67].

\section{Intra-tumoral and local drug delivery}

Anticancer drugs should be delivered at the tumor site inappropriate concentration, for example, paclitaxel loaded microspheres. Filmforming polymers are used to sustain the release at local site, that is, oral cavity [68].

\section{Colonic drug delivery}

Microspheres are used for delivering the drug at a specific site in intestine, that is, colon. Insulin loaded into chitosan microspheres targeted to release its drug at colon [69].

\section{Vaginal drug delivery}

Microspheres drug delivery used for treating vaginal infections such as mycotic infection of the genital tract. Chitosan, Gelatin, and PLGA polymers are used for fabricating the microspheres to treat vaginal infections [70].

\section{Radioactive application}

Radioactive isotopes of elements have been utilized for medical use from decades [71-83] radioactive isotopes loaded microspheres are used

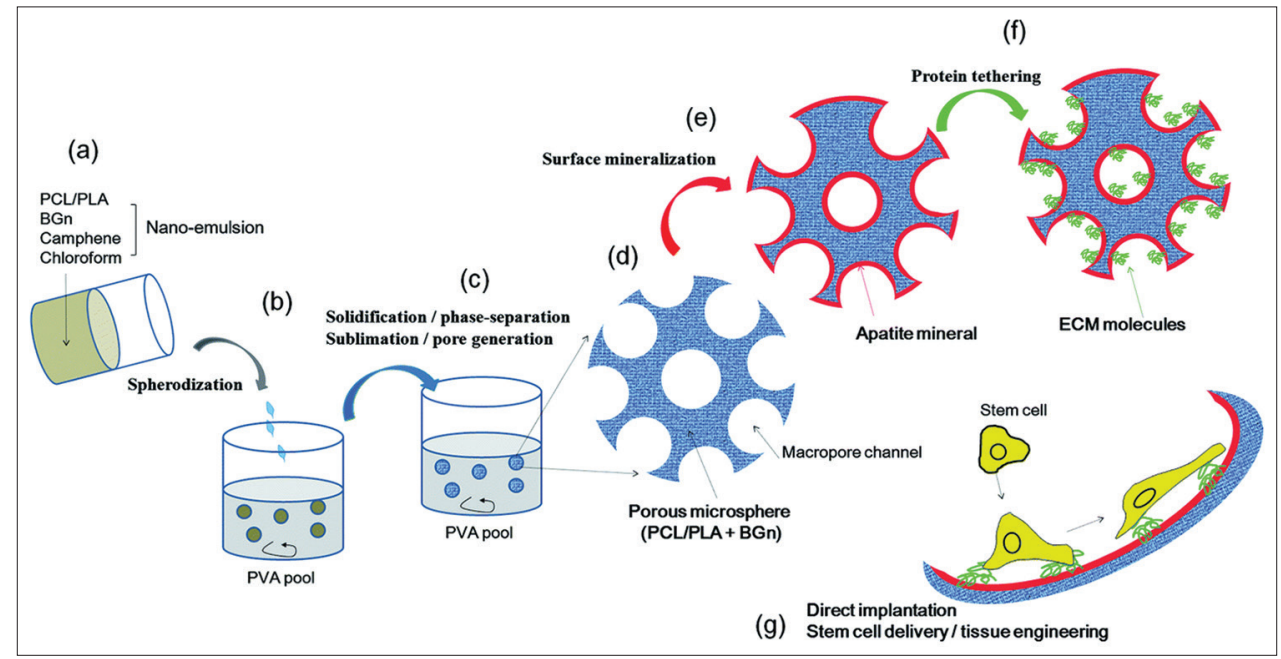

Fig. 3: Microspheres application in bone tissue engineering. Adapted from reference [56]. Copyright permission obtained under Springer Copyright license through Springer Copyright Clearance center 
to treat several diseases such as liver and spleen tumor, for example, Yttrium 90 loaded microspheres are used to treat carcinoma [84].

\section{Vaccine drug delivery}

Microsphere drug delivery has been showing efficient in vaccine delivery; Thiolated Eudragit Microspheres would be a better candidate for oral vaccine delivery. It also evokes the systemic and mucosal immunity [57].

\section{Targeting using microparticulate drug carriers}

Targeting refers to the response elicited by the drug is dependent on the access and molecular interaction with the receptor. Pellets method is generally used to target a particular organ or receptor. Pellets can be prepared by Extrusion/Spheronization Technology, for example, Chitosan and Microcrystalline Cellulose [57].

\section{Application in dentistry}

Microspheres are used in the dental preparations to treat various infections related to oral cavity such as Gingivitis and bleeding gums. Microspheres are also used in craniofacial tissue regeneration [85,87-89].

\section{Application in bone tissue engineering}

Microspheres can be used as a stable carrier system for delivering MSC (Bone marrow-derived mesenchymal stem cell) which helps in earlier bone regeneration as well as rapid bone formation in tissue engineering $[86,90]$. This process is represented diagrammatically as shown in Fig. 3.

\section{CONCLUSIONS}

Microspheres are a type of novel drug delivery system where the drug is enclosed in spherical shaped structures which are made up of different polymers forming a matrix system. The drug liberates out of the microspheres by slow release through the matrix system. Various types of microspheres are available depending on the types of polymers employed and each having their own unique features. There are also many methods of preparation available for the synthesis of microspheres. Microspheres are very versatile and are being used in various applications ranging from diagnostics to drug delivery and medical applications.

\section{ACKNOWLEDGMENT}

The authors are thankful toward the staff of H.K College of Pharmacy for guiding during the preparation of this manuscript.

\section{AUTHORS CONTRIBUTION}

Manuscript framing and the concept has been presented by Mousami Samanta. Literature search and preparation have been done by Deepak Gautam, Muhammed Wasim Chandel and Kirti Sharma. Reviewing and editing have been done by Gaurang Sawant.

\section{CONFLICT OF INTERESTS}

The authors have declared no conflicts of interest.

\section{AUTHORS FUNDING}

As this is a review article, no funding was provided for this study.

\section{REFERENCES}

1. Kakar S, Jain A. Magnetic microspheres: An Overview. Asian Pac J Health Sci 2019;6:81-9.

2. Sharma M, Dev SK, Kumar M, Shukla AK. Microspheres as a suitable drug carrier in sustained release drug delivery: An overview. Asian J Pharm Pharmacol 2018;4:102-8.

3. Nidhi P, Anamika C, Twinkle S, Mehul S, Hitesh J, Umesh U. Controlled drug delivery system: A review. Indo Am J Pharm Sci 2016;3:227-33.

4. Prasad BS, Gupta VR, Devanna N, Jayasurya K. Microspheres as drug delivery system-a review. J Glob Trends Pharm Sci 2014;5:1961-72.

5. Virmani T, Gupta J. Pharmaceutical application of microspheres: An approach for the treatment of various diseases. Int J Pharm Sci Res 2017;8:3252-60.

6. Lengyel M, Kállai-Szabó N, Antal V, Laki AJ, Antal I. Microparticles, microspheres, and microcapsules for advanced drug delivery. Sci Pharm 2019;87:20

7. Farraj NF, Johansen BR, Davis SS, Illum L. Nasal administration of insulin using bioadhesive microspheres as a delivery system. J Control Release 1990;13:253-61.

8. Genta I, Conti B, Perugini P, Pavanetto F, Spadaro A, Puglisi G. Bioadhesive microspheres for ophthalmic administration of acyclovir. J Pharm Pharmacol 1997; 49:737-42.

9. Chandna A, Batra D, Kakar S, Singh R. A review on target drug delivery: Magnetic microspheres. J Acute Dis 2013;2:189-95.

10. Zhang J, Zhang S, Wang Y, Zeng J. Composite magnetic microspheres: Preparation and characterization. J Magn Magn Mater 2007;309:197-201.

11. Sangale SB, Barhate SD, Jain BV, Potdar M. Formulation and evaluation of floating felodipine microsphere. Int J Pharm Res Dev 2011;3:163-70.

12. Srivastava AK, Ridhurkar DN, Wadhwa S. Floating microspheres of cimetidine: Formulation, characterization and in vitro evaluation. Acta Pharm 2005;55:277-85.

13. De Cuyper M, Bulte JW, editors. Urs HÄfeli. In: Radioactive Microspheres for Medical Applications. Physics and Chemistry Basis of Biotechnology. Vol. 7. Springer: Dordrecht; 2001. p. 213-48.

14. El-Helw AM, Al-Hazimi AM, Youssef RM. Preparation of sustained release phenobarbitone microspheres using natural and synthetic polymers. Med Sci 2008;15:39-51.

15. Cai Y, Chen Y, Hong X, Liu Z, Yuan W. Porous microsphere and its applications. Int J Nanomed 2013;8:1111.

16. Zhang CZ, Niu J, Chong YS, Huang YF, Chu Y, Xie SY, et al. Porous microspheres as promising vehicles for the topical delivery of poorly soluble asiaticoside accelerate wound healing and inhibit scar formation in vitro and in vivo. Eur J Pharm Biopharm 2016;109:1-3.

17. Budov VV. Hollow glass microspheres. Use, properties, and technology. Glass Ceram 1994;51:230-5.

18. Li S, Nguyen L, Xiong H, Wang M, Hu TC, She JX, et al. Porouswall hollow glass microspheres as novel potential nanocarriers for biomedical applications. Nanomed Nanotechnol 2010;6:127-36.

19. Ratnaparkhi M, Wattamwar M, Jadhav A, Chaudhari S. Mucoadhesive microsphere-review. Int J Drug Dev Res 2014;6:975-1344.

20. Degim IT, Çelebi N. Controlled delivery of peptides and proteins. Curr Pharm Des 2007;13:99-117.

21. Bansal H, Kaur S, Gupta A. Microsphere: Methods of prepration and applications; a comparative study. Int J Pharm Sci Rev Res 2011;10:69-78.

22. Wu L, Wang M, Singh V, Li H, Guo Z, Gui S, et al. Three-dimensional distribution of surfactant in microspheres revealed by synchrotron radiation X-ray microcomputed tomography. Asian J Pharm Sci 2017;12:326-34.

23. Avachat A, Bornare P, Dash R. Sustained release microspheres of ropinirole hydrochloride: Effect of process parameters. Acta Pharm 2011;61:363-76.

24. Ranjha NM, Khan H, Naseem S. Encapsulation and characterization of controlled release flurbiprofen loaded microspheres using beeswax as an encapsulating agent. J Mater Sci Mater Med 2010;21:1621-30.

25. Pachuau L, Mazumder B. A study on the effects of different surfactants on ethyl cellulose microspheres. Int J Pharm Tech Res 2009;1:966-71.

26. Kim JC, Song ME, Lee EJ, Park SK, Rang MJ, Ahn HJ. Preparation of microspheres by an emulsification-complexation method. J Colloid Interface Sci 2002;248:1-4

27. De Rosa G, Iommelli R, La Rotonda MI, Miro A, Quaglia F. Influence of the co-encapsulation of different non-ionic surfactants on the properties of PLGA insulin-loaded microspheres. J Control Release 2000;69:283-95.

28. Gaur PK, Mishra S, Bajpai M. Formulation and evaluation of controlledrelease of telmisartan microspheres: In vitro/in vivo study. J Food Drug Anal 2014;22:542-8

29. Dinarvand R, Moghadam SH, Sheikhi A, Atyabi F. Effect of surfactant HLB and different formulation variables on the properties of poly-D, L-lactide microspheres of naltrexone prepared by double emulsion technique. J Microencapsul 2005;22:139-51.

30. Shiga K, Muramatsu N, Kondo T. Preparation of poly (D, L-lactide) and copoly (lactide-glycolide) microspheres of uniform size. J Pharm Pharmacol 1996;48:891-5.

31. Wan LS, Heng PW, Chan LW. Surfactant effects on alginate microspheres. Int J Pharm 1994;103:267-75.

32. Khare P, Jain SK. Influence of rheology of dispersion media in the 
preparation of polymeric microspheres through emulsification method. AAPS Pharm Sci Tech 2009;10:1295-300.

33. Kumbar SG, Kulkarni AR, Aminabhavi TM. Crosslinked chitosan microspheres for encapsulation of diclofenac sodium: Effect of crosslinking agent. J Microencapsul 2002;19:173-80.

34. Gülsu A, Ayhan H, Ayhan F. Preparation and characterization of ketoprofen loaded albumin microspheres. Turk J Biochem 2012;37:120-8.

35. Jayan SC, Sandeep AV, Rifash M, Mareema CM, Shamseera S. Design and in vitro evaluation of gelatin microspheres of salbutamol sulphate. Hygeia 2009;1:17-20.

36. Wei W, Wang LY, Yuan L, Wei Q, Yang XD, Su ZG, Ma GH. Preparation and application of novel microspheres possessing autofluorescent properties. Adv Func Mater 2007; 17:3153-8.

37. Behera AL, Patil SV, Sahoo SK. Formulation and characteristics of 5 fluorouracil microspheres by solvent evaporation method. Int J Pharm Pharm Sci 2011;3:32-5.

38. Kendre P, Chaudhari P. Formulation and evaluation of telmisartan microspheres by solvent evaporation technique. Indo Am J Pharm Res 2012;2:651-7.

39. Uyen NT, Hamid ZA, Tram NX, Ahmad N. Fabrication of alginate microspheres for drug delivery: A review. Int J Biol Macromol 2020;153:1035-46

40. Uyen NT, Hamid ZA, Ahmad NB. Synthesis and characterization of curcumin loaded alginate microspheres for drug delivery. J Drug Deliv Sci Technol 2020;58:101796.

41. Mua L, Fenga SS. Fabrication, characterization and in vitro release of paclitaxel (TaxolÒ) loaded poly (lactic-co-glycolic acid) microspheres prepared by spray drying technique with lipid/cholesterol emulsifiers. J Control Release 2001;76:239-54.

42. Zalloum NL, de Souza GA, Martins TD. Single-emulsion P (HB-HV) microsphere preparation tuned by copolymer molar mass and additive interaction. ACS Omega 2019;4:8122-35.

43. Yanga YY, Chiab HH, Chunga TS. Effect of preparation temperature on the characteristics and release profiles of PLGA microspheres containing protein fabricated by double-emulsion solvent extraction/ evaporation method. J Control Release 2000;69:81-96.

44. Bhattacharya S, Alam M, Dhungana K, Yadav S, Chaudhary KR, Chaturvedi KK, et al. Preparation and evaluation of diclofenac gelatin microspheres using coacervation technique. Int $\mathrm{J}$ Pharm Res Innov 2020;13:14-21

45. Bertoni S, Albertini B, Passerini N. Different BCS Class II druggelucire solid dispersions prepared by spray congealing: Evaluation of solid state properties and in vitro performances. Pharmaceutics 2020;12:548.

46. Gurung BD, Kakar S. An overview on microspheres. Int J Health Clin Res 2020;3:11-24.

47. Baimark Y, Srisuwan Y. Preparation of polysaccharide-based microspheres by a water-in-oil emulsion solvent diffusion method for drug carriers. Int J Polym Sci 2013;2013:1-6.

48. Kim JU, Shahbaz HM, Lee H, Kim T, Yang K, Roh YH, et al. Optimization of phytic acid-crosslinked chitosan microspheres for oral insulin delivery using response surface methodology. Int J Pharm 2020;588:119736.

49. Mathiowitz E, Langer R. Polyanhydride microspheres as drug carriers I. Hot-melt microencapsulation. J Control Release 1987;5:13-22.

50. Khanam N, Alam MI, Sachan AK, Gangwar SS. Fabrication and evaluation of propranolol hydrochloride loaded microspheres by ionicgelation technique. Pharm Lett 2012;4:815-20.

51. Patel N, Lalwani D, Gollmer S, Injeti E, Sari Y, Nesamony J. Development and evaluation of a calcium alginate based oral ceftriaxone sodium formulation. Prog Biomater 2016;5:117-33.

52. Fujii S, Okada M, Sawa H, Furuzono T, Nakamura Y. Hydroxyapatite nanoparticles as particulate emulsifier: Fabrication of hydroxyapatitecoated biodegradable microspheres. Langmuir 2009;25:9759-66.

53. Trivedi P, Verma AM, Garud N. Preparation and characterization of aceclofenac microspheres. Asian J Pharm 2014;2:110-5.

54. Pradeesh TS, Sunny MC, Varma HK, Ramesh P. Preparation of microstructured hydroxyapatite microspheres using oil in water emulsions. Bull Mater Sci 2005;28:383-90.

55. Naveen HP, Nesalin JA, Mani TT. A modern review on microsphere as novel controlled drug delivery system. Asian J Res Pharm Sci Biotechnol 2014;2:62-9.

56. Thiel MA, Morlet N, Schulz D, Edelhauser HF, Dart JK, Coster DJ, et al. A simple corneal perfusion chamber for drug penetration and toxicity studies. Br J Ophthalmol 2001;85:450-3.

57. Rastogi V, Shukla SS, Singh R, Lal N, Yadav P. Microspheres: A promising drug carrier. J Drug Deliv Ther 2016;6:18-26.
58. Remuñán-López C, Portero A, Vila-Jato JL, Alonso MJ. Design and evaluation of chitosan/ethylcellulose mucoadhesive bilayered devices for buccal drug delivery. J Control Release 1998;55:143-52.

59. Masaeli R, Kashi TS, Dinarv R, Tahriri M, Rakhshan V, EsfandyariManesh M. Preparation, characterization and evaluation of drug release properties of simvastatin-loaded PLGA microspheres. Iran J Pharm Res 2016;15:205-11.

60. Tejash P, Shah CN, Shah DP. Microspheres: As a novel controlled drug delivery system a review. Pharm Sci Monit 2016;7:37-53.

61. Rathbone MJ. Human buccal absorption. I. A method for estimating the transfer kinetics of drugs across the human buccal membrane. Int $\mathrm{J}$ Pharm 1991;69:103-8.

62. Jiang WZ, Cai Y, Li HY. Chitosan-based spray-dried mucoadhesive microspheres for sustained oromucosal drug delivery. Powder Technol 2017;312:124-32.

63. Liu W, Lee BS, Mieler WF, Kang-Mieler JJ. Biodegradable microspherehydrogel ocular drug delivery system for controlled and extended release of bioactive aflibercept in vitro. Curr Eye Res 2019;44:264-74.

64. Sahu Y, Jain S, Shukla K. Mucoadhesive microspheres based formulation development of ziprasidone hydrochloride for nasal delivery. J Drug Deliv Ther 2020;10:175-81.

65. Xu Q, Leong J, Chua QY, Chi YT, Chow PK, Pack DW, et al. Combined modality doxorubicin-based chemotherapy and chitosan-mediated p53 gene therapy using double-walled microspheres for treatment of human hepatocellular carcinoma. Biomaterials 2013;34:5149-62.

66. Lee S, McAuliffe DJ, Kollias N, Flotte TJ, Doukas AG. Photomechanical delivery of 100-nm microspheres through the stratum corneum: Implications for transdermal drug delivery. Lasers Surg Med 2002;31:207-10.

67. Goswami N, Joshi G, Sawant K. Floating microspheres of valacyclovir $\mathrm{HCl}$ : Formulation, optimization, characterization, in vitro and in vivo floatability studies. J Pharm Bioallied Sci 2012;4 Supp 1:S8-9.

68. Almond BA, Hadba AR, Freeman ST, Cuevas BJ, Yorka AM, Detrisacb CJ, et al. Efficacy of mitoxantrone-loaded albumin microspheres for intratumoral chemotherapy of breast cancer. J Control Release 2003;91:147-55.

69. Wang QS, Wang GF, Zhou J, Gao LN, Cui YL. Colon targeted oral drug delivery system based on alginate-chitosan microspheres loaded with icariin in the treatment of ulcerative colitis. Int J Pharm 2016;515:176-85.

70. Yang TT, Cheng YZ, Qin M, Wang YH, Yu HL, Wang AL, et al. Thermosensitive chitosan hydrogels containing polymeric microspheres for vaginal drug delivery. Biomed Res Int 2017;2017:3564060.

71. Didi A, Dadouch A, El Bekkouri H. Feasibility study for production of iodine-131 using dioxide of tellurium-130. Int J Pharm Pharm Sci 2016;8:e331.

72. Taïeb D, Guille DA, Mundler LO. Guidelines for radionuclide imaging of phaeochromocytoma and paraganglioma. J Med Nucl 2008;32:101-10.

73. Vitaux F. Thyroid gland irradiations and thyroid cancers critical bibliographic journal. J Med Nucl 2007;31:350-5.

74. El Bez I. Cancer de la thyroïde et ablation par iode 131 sous thyrogen: Quand doser la thyroglobuline? Ann Endocrinol 2013;74:156.

75. Spagnoli V, Azzalini L, Tadros VX, Picard F, Ly HQ. Contrast-induced nephropathy: An update. Ann Cardiol Angeiol 2016;65:87-94.

76. Guerrouj H, Elamrani M, Ghfir I, Rais NB. Apport de l'iode 131 dans le traitement de l'adénome thyroïdien toxique. J Med Nucl 2012;36:561-4.

77. Delmaire C. Imagerie des métastases cérébrales. Cancer Radiothér 2015;19:16-9

78. Shah B. Composites from agricultural detritus for pollution remedy. Int J Pharm Pharm Sci 2016;3:4-49.

79. Boisserie G, Hasboun D. Utilisation de l'imagerie multimodalité en radiothérapie. Cancer Radiothé 2001;5:15-35.

80. Belkacémi Y, Tsoutsou PG, Comet B, Kerrou K, Lartigau E. Évaluation de la radiosensibilité tumorale par l'imagerie fonctionnelle et métabolique: De la recherche à l'application clinique. Revue de la littérature. Cancer Radiothér 2006;10:124-33.

81. Mbodj M, Guerrouj H, Amjad I, Rais NA. Contribution of radio-iodine 131 in the treatment of Grave's Basedow disease in the department of nuclear medicine of Ibn sina hospital in Rabat. J Med Nucl 2009;33:592-8.

82. Schlienger JL, Goichot B, Grunenberge F. Iode et fonction thyroïdienne. Rev Méd Int 1997;9:709-16.

83. Hamed MA, Ghany AF, Osman NM. The diagnostic usefulness of FDG-PET/CT in detecting tumor recurrence not evident in whole body I-131 scan in differentiated thyroid carcinoma. Egypt J Radiol Nucl Med 2014;45:361-5.

84. Eyles JE, Spiers ID, Williamson ED, Alpar HO, Williamson ED. Tissue distribution of radioactivity following intranasal administration of radioactive microspheres. J Pharm Pharmacol 2001;53:601-7. 
85. Nafea EH, El-Massik MA, El-Khordagui LK, Marei MK, Khalafallah NM. Alendronate PLGA microspheres with high loading efficiency for dental applications. J Microencapsul 2007;24:525-38.

86. Kanafi MM, Ramesh A, Gupta PK, Bhonde RR. Dental pulp stem cells immobilized in alginate microspheres for applications in bone tissue engineering. Int Endod J 2014;47:687-97.

87. Chang B, Ahuja N, Ma C, Liu X. Injectable scaffolds: Preparation and application in dental and craniofacial regeneration. Mater Sci Eng R Rep 2017;111:1-26.

88. Keskar M, Sabatini C, Cheng C, Swihart MT. Synthesis and characterization of silver nanoparticle-loaded amorphous calcium phosphate microspheres for dental applications. Nano Adv 2019;1:627-35.

89. Zhao XH, Tay FR, Fang YJ, Meng LY, Bian Z. Topical application of phenytoin or nifedipine-loaded PLGA microspheres promotes periodontal regeneration in vivo. Arch Oral Biol 2019;97:42-51.

90. Park JH, Kim MK, Fiqi AE, Seo SJ, Lee EJ, Kim JH, et al. Bioactive and porous-structured nanocomposite microspheres effective for cell delivery: A feasibility study for bone tissue engineering. RSC Adv 2014;4:29062-71. 